\title{
Fitossociologia de florestas de mangue plantadas e naturais no estuário do Rio das Ostras, Rio de Janeiro, Brasil
}

\author{
Elaine Bernini ${ }^{1 *}$ \\ Cristina de Fátima Nunes do Rosário dos Santos ${ }^{2}$ \\ Frederico Lage-Pinto ${ }^{2}$ \\ Gabriele Paiva Chagas ${ }^{2}$ \\ Carlos Eduardo Rezende ${ }^{2}$

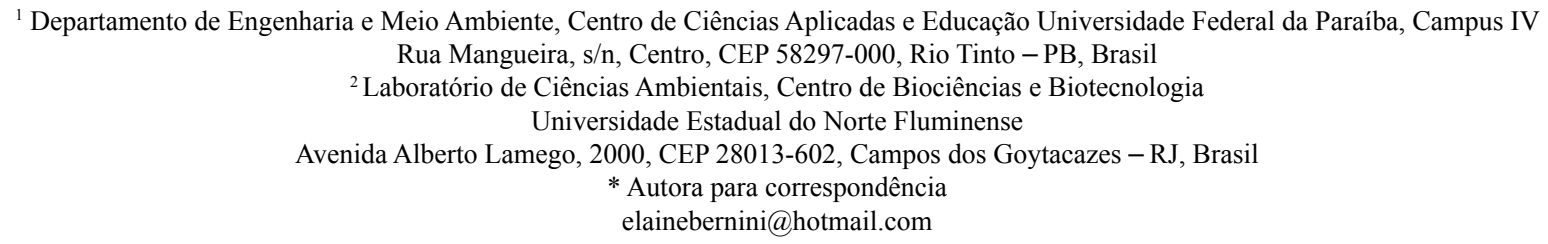

Submetido em 28/05/2013

Aceito para publicação em 09/10/2013

\section{Resumo}

A fitossociologia de florestas de mangue plantadas e naturais foi comparada no estuário do Rio das Ostras, Rio de Janeiro, Brasil. A amostragem da vegetação foi feita pelo método de parcelas, sendo registrado o diâmetro à altura do peito (DAP) e a altura dos indivíduos $\geq 1 \mathrm{~m}$ de altura. Os resultados indicaram que a floresta plantada apresenta menores valores de DAP médio e área basal, e maior densidade de troncos em relação à floresta natural. A distribuição de indivíduos por classes de altura e a distribuição de troncos por classes diamétricas mostraram que a floresta plantada é mais jovem. Laguncularia racemosa e Rhizophora mangle ocorreram em ambas as florestas, enquanto Avicennia schaueriana foi registrada apenas na floresta plantada. Laguncularia racemosa apresentou maior dominância e densidade relativa em todos os sítios analisados, provavelmente, por ser característica de locais com menor influência marinha e pelo fato do estuário ter sido alterado por perturbações antrópicas.

Palavras-chave: Degradação ambiental; Ecossistema estuarino-costeiro; Espécies de mangue; Estrutura de comunidade; Restauração de manguezais

\section{Abstract}

Phytosociology of planted and natural mangrove forests in the estuary of the Ostras River, Rio de Janeiro State, Brazil. The phytosociology of planted and natural mangrove forests were compared in the estuary of the Ostras River, Rio de Janeiro State, Brazil. Vegetation sampling was performed by the plot method, and the diameter at breast height $(\mathrm{DBH})$ and height of individuals $\geq 1 \mathrm{~m}$ tall were recorded. The results indicated that the planted forest had lower average DBH and basal area and higher density of trunks in relation to natural forest. The distribution of individuals by height class and the distribution of stems per diameter class showed that the planted forest was younger. Laguncularia racemosa and Rhizophora mangle occurred in both forests, while 
Avicennia schaueriana was found only in the planted forest. Laguncularia racemosa showed greater dominance and relative density at all sites analyzed, probably because it is characteristic of sites with less marine influence and the fact that the estuary had been altered by human disturbance.

Key words: Environmental degradation; Estuarine-coastal ecosystem; Community structure; Mangrove restoration; Mangrove species; Vegetation structure

\section{Introdução}

O manguezal é um ecossistema costeiro que ocorre ao longo de regiões tropicais e subtropicais, ocupando aproximadamente $137.760 \mathrm{~km}^{2}$ (GIRI et al., 2011). As florestas de mangue estão entre as mais produtivas dos trópicos (DONATO et al., 2011) e desempenham papel fundamental tanto por servir como habitat para diversos tipos de organismos, quanto por fornecer bens e serviços, tais como produção pesqueira, proteção natural contra perturbações e ciclagem de nutrientes (ALONGI, 2008; BARBIER et al., 2008). No entanto, o declínio das áreas de manguezal tem atingido taxas alarmantes nas últimas décadas (GIRI et al., 2011), como resultado da expansão urbana, carcinicultura, atividades industriais, entre outras atividades antropogênicas (ALONGI, 2002; DUKE et al., 2007).

Diante deste cenário, estudos relacionados à recuperação dos manguezais são indispensáveis para amenizar e reverter o quadro de declínio das florestas de mangue. Assim, a recuperação desse ecossistema tem sido descrita em vários estudos, tanto no Brasil (ex. MOSCATELLI et al., 1997; MENEZES et al., 1998; TOGNELLA-DE-ROSA et al., 2002; MENEZES et al., 2005) bem como em outros países (ex. CHAN, 1997; DUKE, 1997; HONG, 1997; PADRÓN, 1997; UNTAWALE, 1997; PROFFITT; DEVLIN, 2005; LUO et al., 2010). Entretanto, existem reduzidas informações sobre o desempenho das florestas plantadas em comparação com as florestas naturais (ex. PROFFITT; DEVLIN, 2005; LUO et al., 2010), havendo a necessidade de estudos de longo prazo e estratégias de plantio com o propósito de analisar se as florestas plantadas atingem a mesma complexidade estrutural das florestas naturais. No Brasil, menos de $0,01 \%$ de toda a área de manguezal já perdida foi restaurada e as experiências conduzidas resultaram em baixas taxas de sobrevivência (ROVAI, 2012).
O manguezal do Rio das Ostras, localizado no Estado do Rio de Janeiro, apresenta-se bastante alterado, pois sofreu um descontrolado processo de urbanização em ambas as margens do rio. Para reverter a situação, a Prefeitura Municipal de Rio das Ostras, por meio da Secretaria Municipal de Meio Ambiente, Agricultura e Pesca e do Comitê de Estudos Ambientais, desapropriou e cercou várias áreas remanescentes para implantar um programa de recuperação e reflorestamento do manguezal, que contou com a participação de escolas, organizações não governamentais, associação de moradores e a comunidade em geral. O programa de recuperação do manguezal vem sendo realizado desde 2003 e se tornou referência na região por ter recuperado áreas de manguezal no município (ENNE, 2010). O programa contemplou eventos de educação ambiental, preservação dos manguezais com remoção de aterro, produção e plantio de mudas de espécies de mangue. Entretanto, o desenvolvimento estrutural das florestas de mangue plantadas ainda não foi avaliado. Dentro deste contexto, o objetivo deste estudo é comparar a fitossociologia entre florestas plantadas e naturais no manguezal do estuário do Rio das Ostras, Rio de Janeiro.

\section{Material e Métodos}

O estudo foi realizado no estuário do Rio das Ostras (Figura 1), localizado no município de Rio das Ostras, Estado do Rio de Janeiro. O município situa-se na região das baixadas litorâneas, entre as coordenadas $22^{\circ} 31^{\prime} 37^{\prime}$ 'S e $41^{\circ} 56^{\prime} 42^{\prime \prime} O$. O clima é tropical, quente e úmido. A precipitação pluviométrica anual varia de 1.120 a $1.180 \mathrm{~mm}$ e a temperatura média anual fica entre 20 e $25^{\circ} \mathrm{C}$ (PRIOSTE, 2007). O Rio das Ostras nasce de dois afluentes principais chamados Rio Iriri e Rio Maurício, com o nome Rio Jundiá, percorre $29 \mathrm{~km}$ no sentido noroeste-sudeste descrevendo uma 
FIGURA 1: Sítios de estudo analisados no manguezal do estuário do Rio das Ostras, Município de Rio das Ostras, RJ. Sítios 1 a 3: florestas plantadas e sítios de 4 a 8: florestas naturais. Fonte: Google Earth Software.

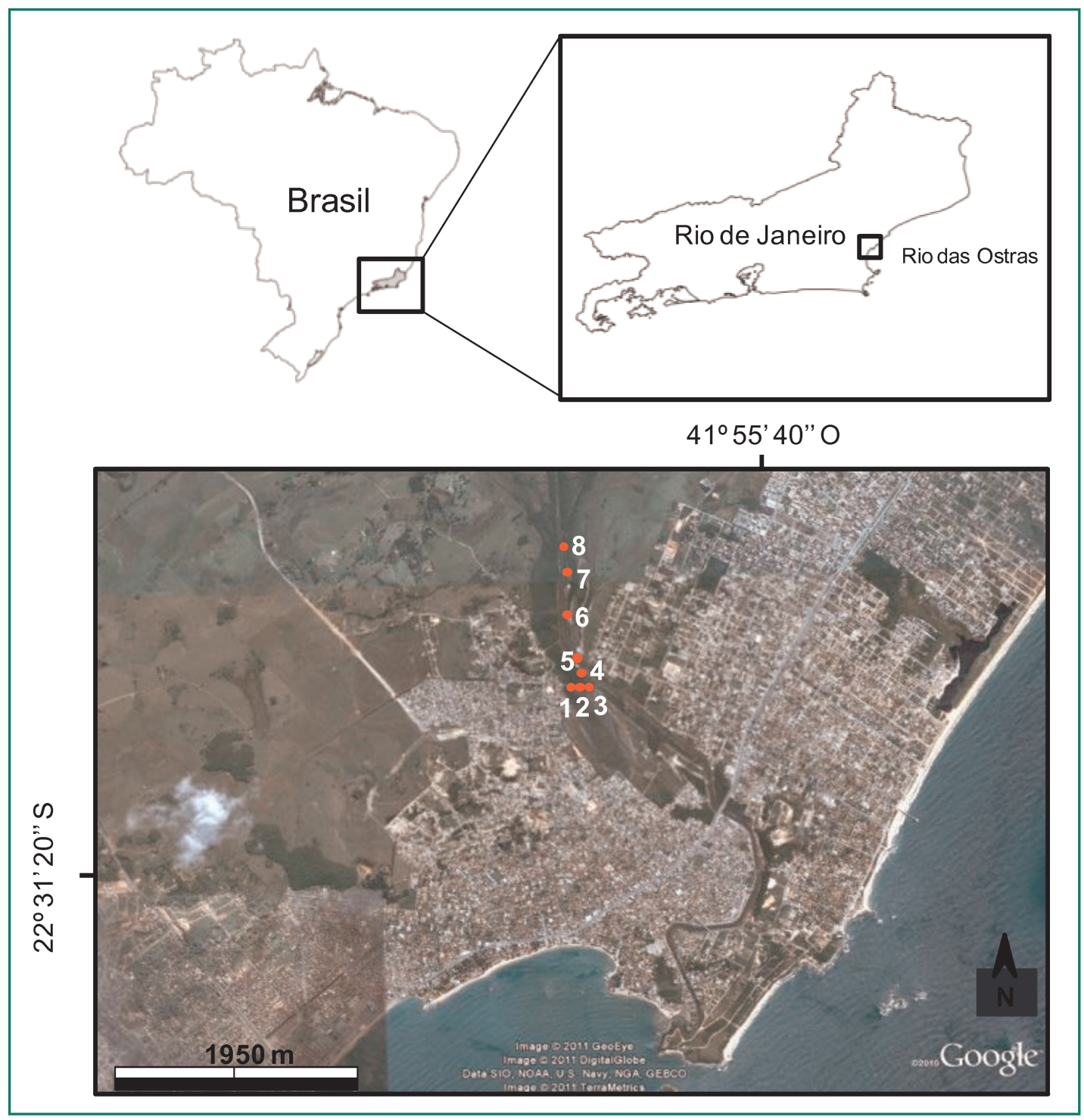

série de meandros até desaguar no Oceano Atlântico. Possui baixa declividade, permitindo a formação de áreas de inundação (PRIMO; VÖLKER, 2003). Devido à baixa declividade, a influência da maré ocorre até $6 \mathrm{~km}$ à montante da foz. Dentro da cidade, o canal tem largura média de $10 \mathrm{~m}$, variando entre 8 a $15 \mathrm{~m}$, com profundidade média de 1,5 a $2,0 \mathrm{~m}$ na preamar. Recebe descarte de dejetos, tais como esgoto doméstico, pneus, lixo plástico e latas. Parte do curso foi canalizado e muitas áreas de várzeas e de mangue foram aterradas para urbanização (PRIMO; VÖLKER, 2003).
A realização do estudo ocorreu no período de março a dezembro de 2010. Para a caracterização da estrutura da vegetação foi utilizado o método de parcelas (SCHAEFFER-NOVELLI; CINTRÓN, 1986). Ao longo do estuário foram selecionados três sítios de estudo (S1, S2 e S3) em áreas de floresta de mangue plantadas (com aproximadamente seis anos de idade de acordo com moradores locais) à margem de um canal artificial e cinco sítios de estudo (S4, S5, S6, S7 e S8) em áreas com florestas naturais de mangue ao longo do rio (Figura 1). A distância entre os sítios na área natural foi maior para haver melhor representatividade do

\section{Demo Version, httpp://www.verydloc.com and htttp://www.verypdif.com}


manguezal. A região da desembocadura (onde também houve plantio de mudas de mangue) não foi amostrada em razão do risco à saúde dos pesquisadores, uma vez que há elevada concentração de lançamento de esgotos domésticos. Além disso, de acordo com moradores locais, nas áreas mais próximas à desembocadura, as mudas de mangue foram plantadas em pontos onde já havia remanescentes de manguezal, não sendo possível definir as áreas como naturais ou plantadas. Por esse motivo, o número de sítios em áreas plantadas foi reduzido.

Em cada sítio foi demarcada uma parcela de $5 \times 10$ $\mathrm{m}$, a $5 \mathrm{~m}$ do canal. $\mathrm{Na}$ área plantada a distância entre as parcelas foi de $10 \mathrm{~m}$ e na área natural a distância variou entre 315 e $630 \mathrm{~m}$. Em cada parcela todas as árvores $\geq 1$ $\mathrm{m}$ de altura foram medidas tanto em altura (com auxílio de vara graduada) quanto em diâmetro à altura do peito (DAP, a 1,3 m do substrato; trena graduada). Indivíduos mortos e ainda em pé foram incluídos na amostragem e são denominados como troncos mortos. As medidas de diâmetro foram agrupadas em classes diamétricas $<$ $2,5 \mathrm{~cm}, \geq 2,5 \mathrm{~cm} \mathrm{e} \geq 10 \mathrm{~cm} \mathrm{e}$, posteriormente, foram calculados os parâmetros DAP médio, altura média, área basal, densidade absoluta, dominância e densidade relativa (SCHAEFFER-NOVELLI; CINTRÓN, 1986). A distribuição de frequência por intervalo de altura e diâmetro foi elaborada para cada floresta analisada. Os dados de DAP médio, altura média, área basal e densidade absoluta foram submetidos ao teste $t$ para verificar diferenças significativas entre as florestas analisadas.

Para caracterizar os fatores abióticos, em cada parcela coletou-se uma amostra composta (três subamostras) de sedimento (15 $\mathrm{cm}$ de profundidade) durante a maré baixa no período seco (junho/2010).
Em laboratório, procedeu-se a remoção de fragmentos grosseiros $>2 \mathrm{~mm}$ com peneira. A salinidade da água intersticial foi determinada com o auxílio de um refratômetro a partir do sobrenadante resultante após a centrifugação de $15 \mathrm{~g}$ de sedimento $(2500 \mathrm{rpm} / 5$ min). A matéria orgânica foi quantificada por meio de incineração em forno mufla $\left(550^{\circ} \mathrm{C} / 1 \mathrm{~h}\right)$ e a granulometria foi determinada em um analisador de partículas (Laser Diffraction, Sald 3101 Shimadzu).

\section{Resultados}

No presente estudo foram registradas as espécies Avicennia schaueriana Stapf \& Leechm. ex Moldenke, Laguncularia racemosa (L.) C. F. Gaertn.f. e Rhizophora mangle L., entretanto, A. schaueriana ocorreu apenas na floresta plantada.

A floresta natural apresentou maiores valores de DAP médio e área basal e menor densidade de troncos em relação à floresta plantada (Tabela 1). A altura das árvores não diferiu significativamente entre as florestas. Houve maior contribuição em área basal na classe entre 2,5 e $10,0 \mathrm{~cm}$ em todos os sítios analisados, sendo registrada área basal $\geq 10,0 \mathrm{~cm}$ apenas nos sítios 7 e 8 (Tabela 2).

A distribuição de indivíduos por classes de altura indicou que ambas as florestas exibiram maior número de indivíduos na classe entre 6,1 e 8,0 m, entretanto, apenas a floresta natural apresentou indivíduos maiores que 8,1 $\mathrm{m}$ (Figura 2). A floresta plantada mostrou maior número de troncos na classe de diâmetro entre 0,1 e 3,0 cm, não ocorrendo indivíduos $>9,0 \mathrm{~cm}$ (Figura 3A), enquanto na floresta natural houve maior número de troncos vivos na classe diamétrica entre 3,1 e $6,0 \mathrm{~cm}$, sendo registrados indivíduos $>9,0 \mathrm{~cm}$ (Figura 3B).

TABELA 1: Parâmetros estruturais da vegetação nas florestas analisadas no manguezal do estuário do Rio das Ostras, RJ. Nas colunas, letras minúsculas comparam os parâmetros estruturais entre as florestas. Letras distintas indicam diferenças significativas para as variáveis entre as duas áreas $(\mathrm{p} \leq 0,05)$. Média \pm desvio padrão; valores entre parênteses representam o coeficiente de variação (\%).

\begin{tabular}{lcccc} 
& $\begin{array}{c}\text { Altura } \\
(\mathbf{m})\end{array}$ & $\begin{array}{c}\text { DAP médio } \\
(\mathbf{c m})\end{array}$ & $\begin{array}{c}\text { Área basal } \\
\left(\mathbf{m}^{\mathbf{2}} \mathbf{h a}^{-\mathbf{}}\right)\end{array}$ & $\begin{array}{c}\text { Densidade } \\
(\text { troncos ha } \mathbf{~})\end{array}$ \\
\hline Floresta plantada & $5,3 \pm 0,2(4) \mathrm{a}$ & $3,0 \pm 0,4(13) \mathrm{b}$ & $25,4 \pm 4,2(17) \mathrm{b}$ & $37.467 \pm 3.349(9) \mathrm{a}$ \\
Floresta natural & $6,8 \pm 1,4(21) \mathrm{a}$ & $5,0 \pm 0,7(14) \mathrm{a}$ & $37,4 \pm 11,8(32) \mathrm{a}$ & $16.540 \pm 11.688(71) \mathrm{b}$ \\
\hline
\end{tabular}


TABELA 2: Área basal $\left(\mathrm{m}^{2} \mathrm{ha}^{-1}\right)$ viva e morta, por classe diamétrica no manguezal do estuário do Rio das Ostras, RJ. Sítios 1 a 3: florestas plantadas e sítios 4 a 8: florestas naturais.

\begin{tabular}{lcccccc}
\hline \multirow{2}{*}{ Sítio } & \multicolumn{3}{c}{ Área basal viva } & \multicolumn{3}{c}{ Área basal morta } \\
\cline { 2 - 7 } & $\mathbf{2}, \mathbf{5} \mathbf{~ c m}$ & $\mathbf{2} \mathbf{5} \mathbf{~ c m}$ & $\mathbf{1 0 , 0} \mathbf{~ c m}$ & $<\mathbf{2 , 5} \mathbf{~ c m}$ & $\mathbf{2} \mathbf{2 , 5} \mathbf{~ c m ~}$ & $\mathbf{\geq 1 0 , 0} \mathbf{~ c m}$ \\
\hline S1 & 2,14 & 20,37 & 0 & 1,27 & 0 & 0 \\
S2 & 1,93 & 21,58 & 0 & 1,44 & 0,75 & 0 \\
S3 & 1,88 & 28,30 & 0 & 2,18 & 0,30 & 0 \\
S4 & 1,32 & 42,15 & 0 & 0,49 & 2,54 & 1,63 \\
S5 & 1,65 & 34,27 & 0 & 0,43 & 0,80 & 0 \\
S6 & 2,11 & 45,14 & 0 & 0,53 & 1,03 & 0 \\
S7 & 0,92 & 38,12 & 3,71 & 2,34 & 2,02 & 0 \\
S8 & 0,14 & 14,04 & 3,43 & 1,19 & 2,63 & 0 \\
\hline
\end{tabular}

FIGURA 2: Distribuição de indivíduos por classes de altura nas florestas analisadas no manguezal do estuário do Rio das Ostras, RJ.

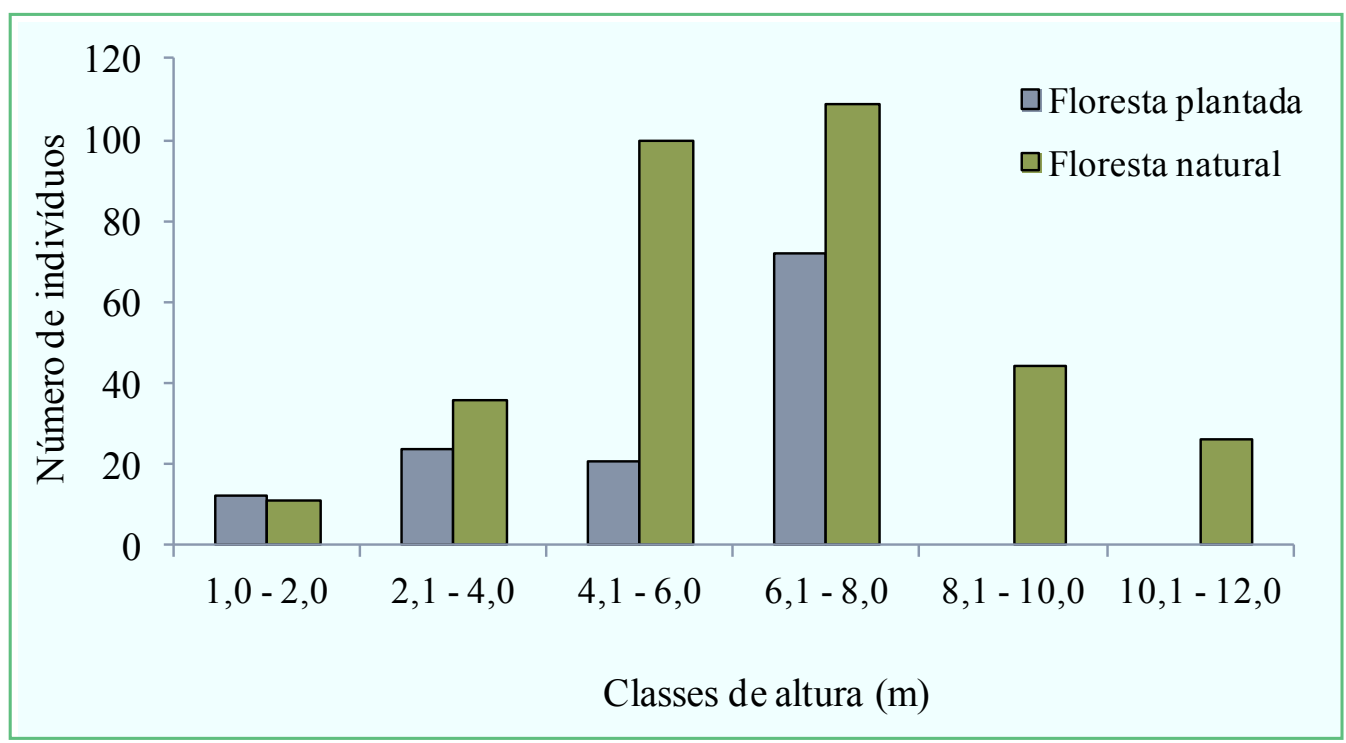


FIGURA 3: Distribuição de troncos por classes de diâmetro nas florestas analisadas no manguezal do estuário do Rio das Ostras, RJ.

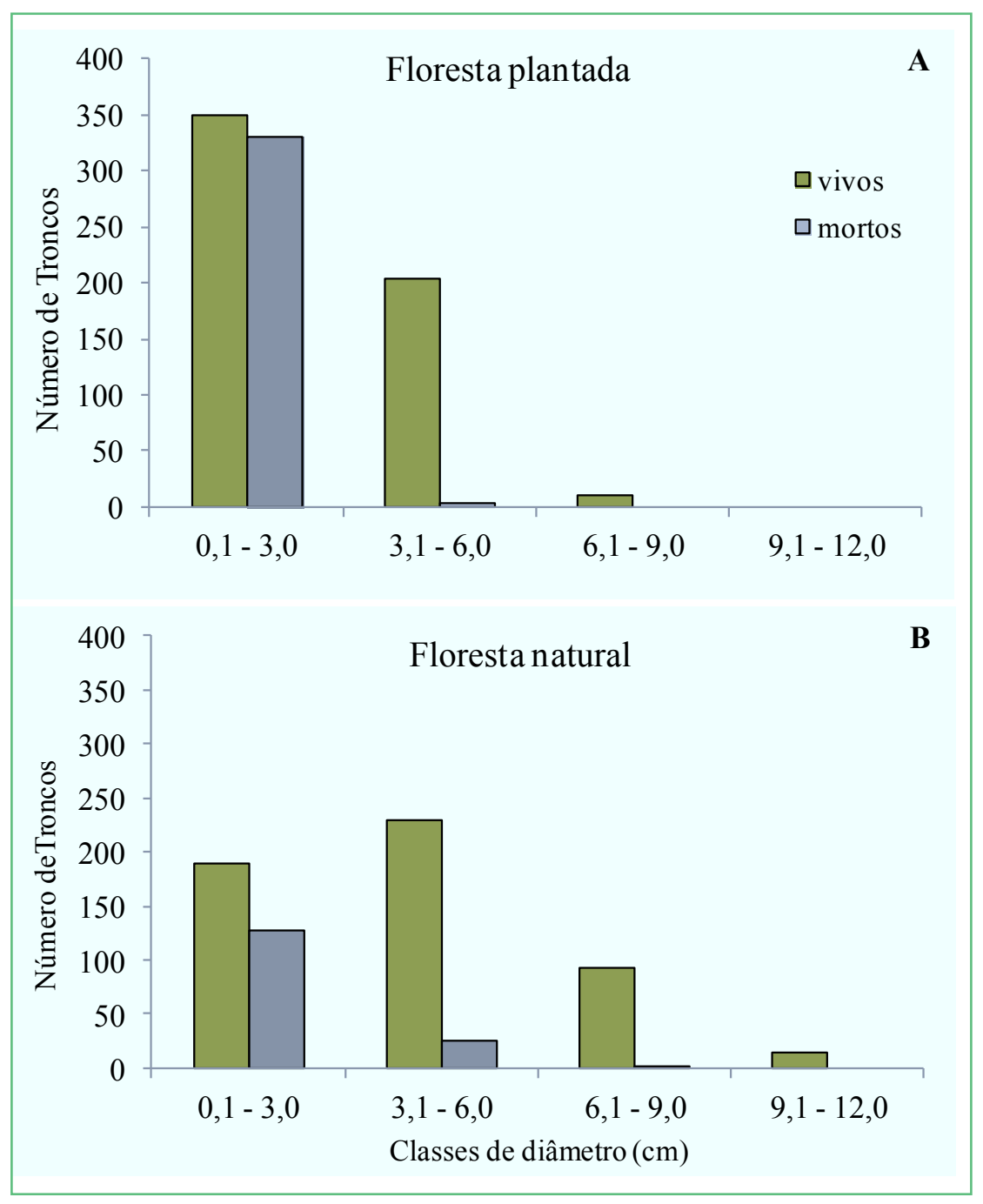

Laguncularia racemosa apresentou maior dominância e densidade relativa em relação às demais espécies tanto na floresta natural, quanto na floresta plantada (Figura 4). Rhizophora mangle exibiu maiores valores de dominância e densidade relativa na floresta plantada em relação à floresta natural, enquanto $A$. schaueriana foi registrada apenas na floresta plantada (Figura 4). Na floresta natural, L. racemosa exibiu maior contribuição em dominância e densidade relativa na classe diamétrica entre 2,5 e $10,0 \mathrm{~cm}$ e $R$. mangle na classe $<2,5 \mathrm{~cm}$ (Tabela 3). Na floresta plantada A. schaueriana e L. racemosa apresentaram maior dominância e densidade relativa na classe diamétrica entre 2,5 e 10,0 cm, e $R$. mangle na classe diamétrica $<$ $2,5 \mathrm{~cm}$. Para troncos mortos, houve maiores valores para L. racemosa, que apresentou maior dominância na classe diamétrica entre 2,5 e 10,0 cm na floresta natural e na classe $<2,5 \mathrm{~cm}$ na floresta plantada. Esta espécie exibiu maior densidade relativa de troncos mortos na classe diamétrica $<2,5 \mathrm{~cm}$ em ambas as florestas (Tabela 3 ). 
FIGURA 4: Dominância (A) e densidade relativa (B) das florestas analisadas no manguezal do estuário do Rio das Ostras, RJ. Sítios 1 a 3: florestas plantadas e sítios 4 a 8: florestas naturais. As: Avicennia schaueriana; Lg: Laguncularia racemosa; Rh: Rhizophora mangle; v: vivos; m: mortos.

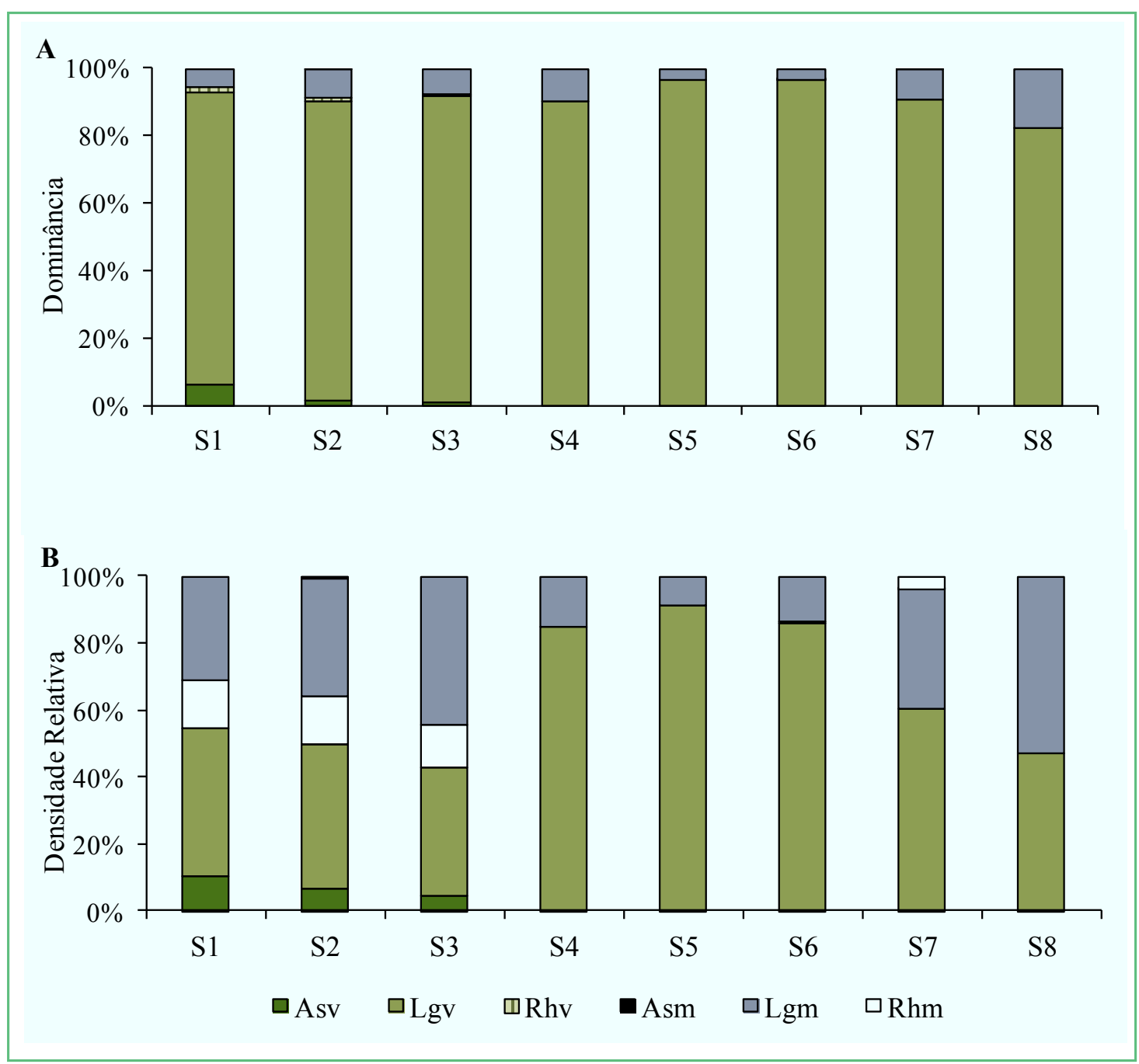


TABELA 3: Dominância (DoA; \%) e densidade relativa (DR; \%) de troncos vivos e mortos, por classe diamétrica e espécie no manguezal do estuário do Rio das Ostras, RJ. Sítios 1 a 3: florestas plantadas e sítios 4 a 8: florestas naturais. As: Avicennia schaueriana; Lg: Laguncularia racemosa; Rh: Rhizophora mangle.

\begin{tabular}{|c|c|c|c|c|c|c|c|c|c|c|c|c|c|c|c|c|c|c|}
\hline & \multicolumn{9}{|c|}{ Troncos Vivos } & \multicolumn{9}{|c|}{ Troncos Mortos } \\
\hline & \multicolumn{3}{|c|}{$<2,5 \mathrm{~cm}$} & \multicolumn{3}{|c|}{$\geq 2,5 \mathrm{~cm}$} & \multicolumn{3}{|c|}{$\geq 10,0 \mathrm{~cm}$} & \multicolumn{3}{|c|}{$<2,5 \mathrm{~cm}$} & \multicolumn{3}{|c|}{$\geq 2,5 \mathrm{~cm}$} & \multicolumn{3}{|c|}{$\geq 10,0 \mathrm{~cm}$} \\
\hline & As & $\mathbf{L g}$ & $\mathbf{R h}$ & As & $\mathbf{L g}$ & $\mathbf{R h}$ & As & $\mathbf{L g}$ & $\mathbf{R h}$ & As & $\mathbf{L g}$ & $\mathbf{R h}$ & As & Lg & $\mathbf{R h}$ & As & Lg & $\mathbf{R h}$ \\
\hline \multicolumn{19}{|c|}{ DoA } \\
\hline S1 & 1,25 & 6,10 & 1,70 & 5,16 & 80,50 & 0 & 0 & 0 & 0 & 0 & 5,30 & 0 & 0 & 0 & 0 & 0 & 0 & 0 \\
\hline S2 & 0,94 & 5,23 & 1,36 & 0,63 & 83,33 & 0 & 0 & 0 & 0 & 0 & 5,58 & 0,02 & 0 & 2,93 & 0 & 0 & 0 & 0 \\
\hline S3 & 0,46 & 4,48 & 0,80 & 0,59 & 86,06 & 0 & 0 & 0 & 0 & 0 & 6,68 & 0 & 0 & 0,93 & 0 & 0 & 0 & 0 \\
\hline S4 & 0 & 2,75 & 0 & 0 & 87,57 & 0 & 0 & 0 & 0 & 0 & 1,01 & 0 & 0 & 5,27 & 0 & 0 & 3,40 & 0 \\
\hline S5 & 0 & 4,44 & 0 & 0 & 92,23 & 0 & 0 & 0 & 0 & 0 & 1,16 & 0 & 0 & 2,17 & 0 & 0 & 0 & 0 \\
\hline S6 & 0 & 4,27 & 0,05 & 0 & 92,48 & 0 & 0 & 0 & 0 & 0 & 1,09 & 0 & 0 & 2,10 & 0 & 0 & 0 & 0 \\
\hline S7 & 0 & 1,94 & 0 & 0 & 80,92 & 0 & 0 & 7,88 & 0 & 0 & 4,78 & 0,18 & 0 & 4,29 & 0 & 0 & 0 & 0 \\
\hline S8 & 0 & 0,64 & 0 & 0 & 65,53 & 0 & 0 & 16,02 & 0 & 0 & 5,53 & 0 & 0 & 12,28 & 0 & 0 & 0 & 0 \\
\hline \multicolumn{19}{|l|}{ DR } \\
\hline S1 & 9,06 & 19,20 & 14,60 & 1,39 & 24,70 & 0 & 0 & 0 & 0 & 0 & 31,00 & 0 & 0 & 0 & 0 & 0 & 0 & 0 \\
\hline S2 & 6,49 & 14,29 & 14,29 & 0,32 & 28,57 & 0 & 0 & 0 & 0 & 0 & 34,74 & 0,32 & 0 & 0,97 & 0 & 0 & 0 & 0 \\
\hline S3 & 4,30 & 10,6 & 12,91 & 0,33 & 27,48 & 0 & 0 & 0 & 0 & 0 & 43,71 & 0 & 0 & 0,66 & 0 & 0 & 0 & 0 \\
\hline S4 & 0 & 28,57 & 0 & 0 & 56,46 & 0 & 0 & 0 & 0 & 0 & 8,84 & 0 & 0 & 5,44 & 0 & 0 & 0,68 & 0 \\
\hline S5 & 0 & 19,21 & 0 & 0 & 72,19 & 0 & 0 & 0 & 0 & 0 & 6,62 & 0 & 0 & 1,99 & 0 & 0 & 0 & 0 \\
\hline S6 & 0 & 23,87 & 0,65 & 0 & 61,94 & 0 & 0 & 0 & 0 & 0 & 9,68 & 0 & 0 & 3,87 & 0 & 0 & 0 & 0 \\
\hline S7 & 0 & 9,15 & 0 & 0 & 50,00 & 0 & 0 & 1,22 & 0 & 0 & 28,66 & 3,66 & 0 & 7,32 & 0 & 0 & 0 & 0 \\
\hline S8 & 0 & 12,50 & 0 & 0 & 31,25 & 0 & 0 & 3,13 & 0 & 0 & 28,13 & 0 & 0 & 25,00 & 0 & 0 & 0 & 0 \\
\hline
\end{tabular}

Os fatores abióticos analisados no sedimento são apresentados na Tabela 4. De modo geral, os valores de salinidade da água intersticial aumentaram na direção rio-mar, variando de 3 a 25 . O percentual de matéria orgânica variou de 6,7 a 23,9\% e a granulometria é tipicamente silte-argilosa em todos os sítios de estudo, com maiores percentuais de silte, seguido por argila e areia.

TABELA 4: Fatores abióticos do sedimento nos sítios analisados no manguezal do estuário do Rio das Ostras, RJ. MO: matéria orgânica. Sítios 1 a 3: florestas plantadas e sítios 4 a 8: florestas naturais.

\begin{tabular}{lccccc}
\hline \multicolumn{1}{c}{ Sítio } & Salinidade & MO (\%) & Areia (\%) & Silte (\%) & Argila (\%) \\
\hline S1 & 10 & 20,5 & 8,9 & 61,7 & 29,4 \\
S2 & 10 & 13,5 & 8,3 & 62,4 & 29,3 \\
S3 & 19 & 10,1 & 9,9 & 64,3 & 25,8 \\
S4 & 25 & 17,2 & 5,2 & 68,8 & 26,0 \\
S5 & 20 & 22,3 & 9,6 & 62,9 & 27,5 \\
S6 & 9 & 6,7 & 7,8 & 68,0 & 24,2 \\
S7 & 5 & 23,9 & 19,6 & 64,6 & 15,8 \\
S8 & 3 & 16,7 & 19,2 & 62,9 & 17,9 \\
\hline
\end{tabular}




\section{Discussão}

Os resultados indicaram que a floresta natural apresentou melhor desenvolvimento estrutural quando comparada à floresta plantada, pois exibiu maiores valores de DAP médio e área basal, além de menor densidade de troncos vivos. Resultados similares foram reportados para uma plantação de $R$. mangle com 11 anos de idade na Flórida, que apresentou maior densidade quando comparada à floresta natural (PROFFITT; DEVLIN, 2005). De acordo com Mckee e Faulkner (2000), o desenvolvimento estrutural de florestas restauradas ou plantadas depende de vários fatores, mas características específicas do local, bem como diferenças regionais na hidrologia e fatores concomitantes, tais como salinidade e grau de inundação, exercem forte influência nos resultados dos projetos de recuperação. Assim, os manguezais podem requerer décadas para atingir a maturidade estrutural (TWILLEY et al., 1998; PROFFITT; DEVLIN, 2005; FERWERDA et al., 2007). Entretanto, dependendo das condições ambientais e das técnicas de restauração, algumas florestas jovens de mangue podem exercer funções importantes na paisagem, mesmo com apenas seis anos de idade (MCKEE; FAULKNER, 2000).

Em um manguezal da China, Luo et al. (2010) observaram que florestas plantadas com 50 anos de idade não diferiram de florestas naturais em relação ao DAP médio, densidade e biomassa aérea, mas verificaram maiores coeficientes de variação para estas variáveis na floresta plantada. De acordo com os autores, estes resultados parecem refletir a alta heterogeneidade espaçotemporal no desenvolvimento e sucessão de florestas plantadas e indiretamente demonstram a sensibilidade da plantação a flutuações nas condições ambientais, tais como distúrbios climáticos e ação de patógenos. No presente estudo, entretanto, foram observados menores valores de coeficiente de variação na floresta plantada para todos os parâmetros estruturais analisados, provavelmente, pelo fato de que as parcelas foram distanciadas em apenas $10 \mathrm{~m}$ entre si, reduzindo assim a variabilidade das características ambientais e refletindo em baixa variabilidade dos parâmetros estruturais. Por outro lado, na floresta natural as parcelas foram mais distanciadas entre si, propiciando maior variabilidade das condições ambientais e, consequentemente, maiores valores de coeficiente de variação dos parâmetros estruturais. Outro fator que também pode ser responsável pela diferença de variabilidade nos parâmetros estruturais entre as florestas é a idade das florestas e o histórico de influência antrópica no estuário. Na floresta plantada, as árvores apresentam idades similares por terem sido plantadas em um mesmo momento, enquanto na floresta natural a idade das árvores é distinta, tanto pelo momento de estabelecimento dos diásporos, como pela influência antrópica (intenso corte de vegetação no passado).

As florestas analisadas no estuário do Rio das Ostras apresentaram maior contribuição na classe diamétrica entre 2,5 e 10,0 cm, indicando baixo desenvolvimento estrutural. Este resultado também foi reportado para outros manguezais sujeitos a elevada influência antrópica, tais como o manguezal da Lagoa da Tijuca, RJ (SOARES 1999) e o manguezal do Rio Guaxindiba, RJ (CHAGAS, 2011). O manguezal do estuário do Rio das Ostras é um dos mais degradados do Estado do Rio de Janeiro, pois sofreu grande perda de área devido ao avanço da urbanização, aterros, corte de vegetação, obras de dragagem e retificações que alteraram a circulação hídrica do ecossistema, resultando em vegetação bastante alterada. Em alguns locais ao longo do estuário é possível observar a mortalidade de árvores devido às alterações na circulação hídrica. Áreas de manguezal com corte de vegetação, aterro ou alagamento podem exibir redução na estatura, diâmetro e área basal (SOUZA; SAMPAIO, 2001).

A distribuição de indivíduos por classes de altura e a distribuição de troncos por classe de diâmetro demonstraram que a floresta plantada (Sítios 1 a 3) apresentou maior contribuição nas menores classes de altura e diâmetro e ausência ou menor contribuição nas maiores classes, provavelmente, por ser mais jovem, pois a floresta tem cerca de seis anos. Luo et al. (2010) também encontraram maior número de árvores com menor porte em florestas plantadas quando comparadas a florestas naturais. No presente estudo, ambas as florestas apresentaram distribuição de troncos por classe de diâmetro do tipo "jota" reverso. De acordo com Soares et al. (2003), isto é indicativo de floresta saudável, pois há mortalidade natural relacionada ao 
amadurecimento da floresta. Este tipo de distribuição é comum em manguezais (JIMENEZ; SAUTER, 1991; SOUZA; SAMPAIO, 2001; SOARES et al., 2003; BERNINI; REZENDE, 2010; 2011; PETRI et al., 2011; CALEGARIO, 2012). Houve maior mortalidade de troncos nos sítios 1, 2, 3, 7 e 8, principalmente de $L$. racemosa. Nos sítios 1 a 3 , onde a floresta é mais jovem, a mortalidade poderia ser atribuída ao desbaste natural resultante da competição intraespecífica, enquanto nos sítios 7 e 8 a mortalidade estaria mais relacionada à senescência da floresta, visto que as florestas desses sítios apresentaram contribuição em área basal na classe de diâmetro $\geq 10,0 \mathrm{~cm}$, sendo mais antigas. Além disso, nos sítios 7 e 8 as cascas dos troncos de L. racemosa são mais rugosas e se soltam mais facilmente, o que é característico de árvores senescentes dessa espécie. A distribuição de troncos por classe de diâmetro permitiu constatar a ausência de corte seletivo nas áreas analisadas (observaram-se apenas algumas árvores cortadas), indicando bom resultado do programa de recuperação do manguezal realizado pela prefeitura do município, que contou com campanhas de educação ambiental.

Em ambas as florestas analisadas L racemosa apresentou maior dominância e maior densidade relativa. De acordo com informações de um servidor da prefeitura, que trabalhou no projeto de restauração do manguezal, as mudas plantadas corresponderam às três espécies que ocorrem no estuário, mas não conseguimos informações sobre a proporção de cada espécie plantada. A maior dominância e densidade relativa de $L$. racemosa na floresta plantada podem estar refletindo as melhores condições ambientais locais para essa espécie e o seu maior recrutamento em função da maior disponibilidade de diásporos provenientes das florestas naturais. Alguns estudos realizados ao longo de gradientes estuarinos demonstraram que $L$. racemosa apresenta maior densidade relativa em locais sob menor influência marinha (SILVA et al., 2005; PETRI et al., 2011; CALEGARIO, 2012; COSTA, 2012), como é o caso do presente estudo. Entretanto, como descrito anteriormente, o manguezal do estuário do Rio das Ostras sofreu muitas ações de degradação, e L. racemosa é característica de florestas perturbadas em processo de regeneração (TOMLINSON, 1986; PERIA et al., 1990;
SOARES, 1999; SOUZA; SAMPAIO, 2001; BERNINI; REZENDE, 2010).

Avicennia schaueriana é característica de locais com maior influência marinha ao longo do gradiente estuarino (SILVA et al., 2005; PETRI et al., 2011; CALEGARIO, 2012; COSTA, 2012), o que poderia explicar sua ausência nas florestas naturais analisadas no presente estudo. De fato, em uma visita à região da desembocadura do Rio das Ostras foi possível observar a predominância de A. schaueriana. Entretanto, como no caso de $L$. racemosa, sua distribuição ao longo do estuário também pode ter sido modificada devido à influência antrópica.

Os resultados demonstraram que a floresta plantada exibiu menor desenvolvimento estrutural quando comparada à floresta natural, por se tratar de uma floresta mais jovem. A riqueza variou, sendo mais elevada na floresta plantada, pois o plantio de mudas realizado pelo programa de recuperação contemplou as três espécies que ocorrem no estuário. Estudos recomendam períodos de 10 a 50 anos (CREWZ; LEWIS, 1991; LUO et al., 2010) para avaliar o sucesso da restauração, baseado nas características estruturais da vegetação. Florestas plantadas podem exercer o mesmo papel ecológico que florestas naturais, dependendo da sua estrutura e função (LUO et al., 2010). Assim, sugerem-se futuros estudos no manguezal do estuário do Rio das Ostras para monitorar tanto o desenvolvimento estrutural, quanto as características funcionais do manguezal (ex. produtividade primária e eficiência fotossintética), com a finalidade de verificar se a floresta plantada exercerá o mesmo papel ecológico que as florestas naturais.

\section{Agradecimentos}

À CAPES, pela bolsa de Pós-Doutorado concedida à primeira autora e pelo apoio ao projeto "Estrutura, Biogeoquímica e Metagenômica de Manguezais" (AUX-PE-PNPD-2303/2008). Aos técnicos de campo e aos motoristas da UENF, pelo apoio nos trabalhos de campo. À Gabriela Calegario, pelo auxílio nas análises de laboratório. Ao Laboratório de Ciências Ambientais do Centro de Biociências e Biotecnologia, pela infraestrutura fornecida para o desenvolvimento do 
trabalho. Este trabalho está inserido no Instituto Nacional de Ciência e Tecnologia - TMCOcean (CNPq Processo 573601/2008-9).

\section{Referências}

ALONGI, D. M. Present state and future of the world's mangrove forests. Environmental Conservation, Cambridge, v. 29, p. 331$349,2002$.

ALONGI, D. M. Mangrove forests: resilience, protection from tsunamis, and responses to global climate change. Estuarine, Coastal and Shelf Science, New York, v. 76, p. 1-13, 2008.

BARBIER, E. B.; KOCH, E. W.; SILLIMAN, B. R.; HACKER, S. D.; WOLANSKI, E.; PRIMAVERA, J.; GRANEK, E. F.; POLASKY, S.; ASWANI, S.; CRAMER, L. A.; STOMS, D. M.; KENNEDY, C. J.; BAEL, D.; KAPPEL, C. V.; PERILLO, G. M. E.; REED, D. J. Coastal ecosystem based management with nonlinear ecological functions and values. Science, London, v. 319, p. 321323, 2008 .

BERNINI, E.; REZENDE, C. E. Variação estrutural em florestas de mangue do estuário do Rio Itabapoana, ES-RJ. Biotemas, Florianópolis, v. 23, p. 49-60, 2010.

BERNINI, E.; REZENDE, C. E. Vegetation structure in a mangrove forest in Southeastern Brazil. Pan-American Journal of Aquatic Sciences, Rio Grande, v. 6, p. 193-209, 2011.

CALEGARIO, G. Aspectos estruturais da vegetação do manguezal do estuário do Rio São João, RJ.2008. 60 f. Dissertação (Mestrado em Ecologia e Recursos Naturais) $\square$ Universidade Estadual do Norte Fluminense, Campos dos Goytacazes. 2012.

CHAGAS, G. P. Estrutura e distribuição das espécies no manguezal do Complexo Rio Guaxindiba/canal Engenheiro Antônio Rezende, São Francisco de Itabapoana, RJ. 2011. 23 f. Monografia (Bacharelado em Biologia) - Universidade Estadual do Norte Fluminense, Campos dos Goytacazes. 2011.

CHAN, H. T. Mangrove reforestation in Peninsular Malaysia: a case study of Matang. In: FIELD, C. D. (Ed.). La restauración de ecosistemas de manglares. Okinawa: Sociedad Internacional para Ecosistemas de Manglar, 1997. p. 64-75.

COSTA, P. C. Estrutura vegetal dos manguezais ao longo de gradientes estuarinos na Baía de Todos os Santos, BA. 2012. 60 f. Dissertação (Mestrado em Ecologia e Biomonitoramento) Universidade Federal da Bahia, Salvador. 2012.

CREWZ, D. W.; LEWIS, R. R. An evaluation of historical attempts to establish emergent vegetation in marine wetlands in Florida. Florida Sea Grant Technical Publication $n^{\circ} 60$. Gainesville: Florida Sea Grant, 1991. 20 p.

DONATO, D. C.; KAUFFMAN, J. B.; MURDIYARSO, D.; KURNIANTO, S.; STIDHAM, M.; KANNINEN, M. Mangroves among the most carbon-rich forests in the tropics. Nature Geoscience, London, v. 4, p. 293-297, 2011.

DUKE, N. Reforestación de manglares en Panamá: una evaluación de la plantación de manglares en áreas desforestadas por un gran derrame de petróleo. In: FIELD, C. D. (Ed.). La restauración de ecosistemas de manglares. Okinawa: Sociedad Internacional para Ecosistemas de Manglar, 1997. p. 231-258.
DUKE, N. C.; MEYNECKE, J. O.; DITTMANN, S.; ELLISON, M.; ANGER, K.; BERGER, U.; CANNICCI, S.; DIELE, K.; EWEL, K. C.; FIELD, D.; KOEDAM, N.; LEE, S. Y.; MARCHAND, C.; NORDHAUS, I.; DAHDOUH-GUEBAS, F. A world without mangroves? Science, London, v. 317, p. 41-42, 2007.

ENNE, E. Por uma região sustentável. Visão Socioambiental, Macaé, v. 22, p. 10-13, 2010.

FERWERDA, J. G.; KETNER, P.; MCGUINNESS, K. A. Differences in regeneration between hurricane damaged and clearcut mangrove stands 25 years after clearing. Hydrobiologia, Brussels, v. 591, p. 35-45, 2007.

GIRI, C.; OCHIENG, E.; TIESZEN, L. L.; ZHU, Z.; SINGH, A.; LOVELAND, T.; MASE, J.; DUKE, N. Status and distribution of mangrove forests of the world using earth observation satellite data. Global Ecology and Biogeography, Ottawa, v. 20, p. 154159, 2011.

HONG, P. N. Restoration of mangrove ecosystems in Vietnam: a case study of Can Gio District, Ho Chi Minh City. In: FIELD, C. D. (Ed.). La restauración de ecosistemas de manglares. Okinawa: Sociedad Internacional para Ecosistemas de Manglar, 1997. p. 7696.

JIMENEZ, J. A.; SAUTER, K. Structure and dynamics of mangrove forests along a flooding gradient. Estuaries, Port Republic, v. 14, n. 1, 49-56, 1991.

LUO, Z.; SUN, O. J.; XU, H. A comparison of species composition and stand structure between planted and natural mangrove forests in Shenzhen Bay, South China. Journal of Plant Ecology, Oxford, v. 3, p. 165-174, 2010.

MCKEE, K. L.; FAULKNER, P. L. Restoration of biogeochemical function in mangrove forests. Restoration Ecology, Crawley, v. 8, p. 247-259, 2000.

MENEZES, G. V.; POFFO, I. R. F.; EYSINK, G. G. J. Estudo sobre a viabilidade e diferentes técnicas de recuperação de manguezais degradados em Cubatão, SP. Relatório Final. São Paulo: CETESB, 1998. 31 p.

MENEZES, G. V.; SCHAEFFER-NOVELLI, Y.; POFFO, I. R. F.; EYSINK, G. G. J. Recuperação de manguezais: um estudo de caso na Baixada Santista de São Paulo, Brasil. Brazilian Journal of Aquatic Science and Technology, Itajaí, v. 9, p. 67-74, 2005.

MOSCATELLI, M.; TEIXEIRA, M. L. F.; ALMEIDA, J. R. O estado da arte na restauração de manguezais no estado do Rio de Janeiro. In: SIMPÓSIO NACIONAL DE RECUPERAÇÃO DE ÁREAS DEGRADADAS, 3, 1997, Ouro Preto. Anais... Ouro Preto. p. 525-534.

PADRÓN, C. M. Restauración de ecosistemas de manglar em Cuba. Estudio de caso de Provincia Habana. In: FIELD, C. D. (Ed.). La restauración de ecosistemas de manglares. Okinawa: Sociedad Internacional para Ecosistemas de Manglar, 1997. p. 176-186.

PERIA, L. C. S.; FERNANDEZ, P. P. C. P.; MENEZES, G. V.; GRASSO, M.; TOGNELLA, M. M. P. Estudos estruturais comparativos entre florestas de mangue impactadas (Canal de Bertioga) e não impactados (Ilha do Cardoso), Estado de São Paulo. In: SIMPÓSIO DE ECOSSISTEMAS DA COSTA SUL E SUDESTE BRASILEIRA, 2, 1990, Águas de Lindóia. Anais... Águas de Lindóia: ACIESP. p. 183-193.

PETRI, D. J. C.; BERNINI, E.; SOUZA, L. M.; REZENDE, C. E. Distribuição das espécies e estrutura do manguezal do Rio 
Benevente, Anchieta, ES. Biota Neotropica, Campinas, v. 11, p. 1-10, 2011.

PRIMO, P. B.; VÖLKER, C. M. Bacias hidrográficas dos rios São João e das Ostras $\square$ águas, terras e conservação ambiental. Rio de Janeiro: CILSJ, 2003. 177 p.

PRIOSTE, M. A. O. Bacia hidrográfica do Rio das Ostras: proposta para gestão ambiental sustentável. 2007. 192 f. Dissertação (Mestrado em Engenharia Ambiental) $\square$ Universidade do Estado do Rio de Janeiro, Rio de Janeiro. 2007.

PROFFITT, C. E.; DEVLIN, D. J. Long-term growth and succession in restored and natural mangrove forests in southwestern Florida. Wetlands Ecology and Management, Baarn, v. 13, p. 531-551, 2005.

ROVAI, A. S. Restauração de manguezais no Brasil: retrospectiva e perspectivas. 2012. 74 f. Dissertação (Mestrado em Ecologia) Universidade Federal de Santa Catarina, Florianópolis. 2012.

SCHAEFFER-NOVELLI, Y.; CINTRÓN, G. Guia para estudo de áreas de manguezal: estrutura, função e flora. São Paulo: Caribbean Ecological Research, 1986. 150 p.

SILVA, M. A. B.; BERNINI, E.; CARMO, T. M. S. Características estruturais de bosques de mangue do estuário do Rio São Mateus, ES, Brasil. Acta Botanica Brasilica, Feira de Santana, v. 19, p. 465-471, 2005.

SOARES, M. L. G. Estrutura vegetal e grau de perturbação dos manguezais da Lagoa da Tijuca, Rio de Janeiro, RJ, Brasil. Revista Brasileira de Biologia, São Paulo, v. 59, p. 503-515, 1999.
SOARES, M. L. G.; CHAVES, F. O.; CORRÊA, F. M.; SILVA-JR., C. M. G. Diversidade estrutural de bosques de mangue e sua relação com distúrbios de origem antrópica: o caso da Baía de Guanabara (Rio de Janeiro). Anuário do Instituto de Geociências, Rio de Janeiro, v. 26, p. 101-116, 2003.

SOUZA, M. M. A.; SAMPAIO, E. V. S. B. Variação temporal da estrutura de bosques de mangue de Suape-PE após a construção do Porto. Acta Botanica Brasilica, Feira de Santana, v. 15, p. 1-12, 2001.

TOGNELLA-DE-ROSA, M. M. P.; OLIVEIRA, R. G.; LUGLI, D. O.; WELLRICH, J. F.; MEIRELES, R. P.; POLLETO, T. V. Replantio da vegetação do ecossistema manguezal do Saco da Fazenda, Itajaí (SC). Notas Técnicas da FACIMAR, Itajaí, v. 6, p. 85-91, 2002.

TOMLINSON, P. B. The botany of mangroves. New York: Cambridge University Press, 1986. 413 p.

TWILlEY, R. R.; RIVERA-MONROY, V. H.; CHEN, R.; BOTERO, R. Adapting an ecological mangrove model to simulate trajectories in restoration ecology. Marine Pollution Bulletin, Amsterdam, v. 37, p. 404-419, 1998.

UNTAWALE, A. G. Restauración de manglares a lo largo de la Costa Oeste Central de La India. In: FIELD, C. D. (Ed.). La restauración de ecosistemas de manglares. Okinawa: Sociedad Internacional para Ecosistemas de Manglar, 1997. p. 121-137.

VALIELA, I.; BOWEN, J. I.; YORK, J. K. Mangrove forests: one of the world's threatened major tropical environments. BioScience, Berkeley, v. 51, p. 807-815, 2001. 